\title{
Order in the chaos? The strange case of accreting millisecond pulsars
}

\author{
Tiziana Di Salvo*, Luciano Burderi ${ }^{\dagger}$, Alessandro Riggio ${ }^{\dagger}$, Alessandro \\ Papitto $^{* *, \sharp}$ and Maria Teresa Menna ${ }^{\ddagger}$ \\ *Dipartimento di Scienze Fisiche ed Astronomiche, Università di Palermo, via Archirafi 36 - 90123 \\ Palermo, Italy \\ ${ }^{\dagger}$ Università degli Studi di Cagliari, Dipartimento di Fisica, SP Monserrato-Sestu, KM 0.7, 09042 \\ Monserrato, Italy \\ ${ }^{* *}$ Dipartimento di Fisica, Universitá degli Studi di Roma 'Tor Vergata', via della Ricerca \\ Scientifica 1, 00133 Roma, Italy \\ ${ }^{\ddagger}$ Osservatorio Astronomico di Roma, Via Frascati 33, 00040 Monteporzio Catone (Roma), Italy
}

\begin{abstract}
We review recent results from the X-ray timing of accreting millisecond pulsars in LMXBs. This is the first time a timing analysis is performed on accreting millisecond pulsars, and for the first time we can obtain information on the behavior of a very fast pulsar subject to accretion torques. We find both spin-up and spin-down behaviors, from which, using available models for the accretion torques, we derive information on the mass accretion rate and magnetic field of the neutron star in these systems. We also find that the phase delays behavior as a function of time in these sources is sometimes quite complex and difficult to interpret, since phase shifts, most probably driven by variations of the X-ray flux, are sometimes present.
\end{abstract}

Keywords: stars: neutron - stars: magnetic fields - pulsars: general - pulsars: individual: SAX J1808.4-3658, IGR J00291+5934, XTE J1814-338, XTE J0929-314 — X-ray: binaries

PACS: 97.60.Jd, 97.80.-d, 97.80.Jp, 97.60.Gb

\section{INTRODUCTION}

According to the recycling scenario (see e.g. [1] for a review), there exists an evolutionary connection between the so-called Low Mass X-ray Binaries (LMXBs) containing a neutron star and Millisecond Radio Pulsars (MSP). The first class of sources consists of old systems where a low magnetized $\left(\sim 10^{8}-10^{9}\right.$ Gauss $)$ neutron star accretes matter from a low mass (usually less or of the order of $\mathrm{M}_{\odot}$ ) companion. The weak magnetic field of the neutron star allows the matter to be accreted very close to the compact object; the accretion radius is indeed the magnetospheric radius (the radius at which the magnetic pressure due to the assumed dipolar magnetic field of the neutron star is balanced by the ram pressure of the accreting matter) which, for typical values of the magnetic field and the mass accretion rate, can be quite close (a few neutron star radii) to the compact object. In this situation, the neutron star can be accelerated by the accretion of matter and angular momentum from a (Keplerian) accretion disk to very short periods, in principle up to the limiting period (usually of the order of or below $1 \mathrm{~ms}$ ), which depends on the mass-radius relation of the neutron star, and therefore on the equation of state of ultra-dense matter. At the end of the mass transfer phase, these systems will be observed as low magnetized, very fast (millisecond) pulsars in a binary system with a 
very low mass (if any) companion star; these systems are indeed observed in radio and form the class of MSP.

This evolutionary scenario was spectacularly confirmed by the discovery of millisecond coherent pulsations in LMXBs; this important discovery arrived recently, in 1998, when coherent millisecond pulsations with a period of $2.5 \mathrm{~ms}$ where discovered in the transient LMXB SAX J1808.4-3658 [2], thanks to the large effective area $\left(\sim 6000 \mathrm{~cm}^{2}\right)$ and high time resolution (up to $1 \mu \mathrm{sec}$ ) of the Proportional Counter Array (PCA) on board the Rossi X-ray Timing Explorer (RXTE). SAX J1808.4-3658, for the rest a quite common LMXB, belongs to a close binary system, $P_{\text {orb }} \simeq 2 \mathrm{~h}$ [3], and is the only one among the known accreting millisecond pulsars which has shown more than one X-ray outburst in the RXTE era. We now know seven accreting millisecond pulsars (see [4], [5] for reviews); all of them are X-ray transients in very compact systems (orbital period between $40 \mathrm{~min}$ and $4 \mathrm{~h}$ ), the fastest of which is IGR J00291+5934, with a spin period of $P_{\text {spin }} \simeq 1.7 \mathrm{~ms}$, and the slowest of which is XTE J0929-314, with a spin period of $P_{\text {spin }} \simeq 5.4 \mathrm{~ms}$.

In this paper we review recent results from timing analysis of a sample of accreting millisecond pulsars. In particular, we first describe the timing technique we have used to analyse these sources, and then we present the results we have obtained to date for four (out of seven) accreting millisecond pulsars.

\section{TIMING ANALYSIS}

The timing analysis we have applied to our sample of accreting millisecond pulsars is based on standard timing techniques that are fully described in [6], and that we briefly describe here.

We use the fact that orbital periods of these systems (typically less than a few hours) are much smaller than the timescale on which intrinsic variations of the spin period are expected to produce their effects on the pulse phase delays (typically tens of days). Also, variations of the phase delays caused by uncertainties in the source position on the sky are expected to show their effects on even longer timescales (a fraction of a year). We therefore proceed in the following way. First of all, the arrival times of all the events are reported to the Solar system barycenter, adopting the best estimate of the source position on the sky. These arrival times are then corrected for the Doppler effects caused by the binary motion using the best estimates of the orbital parameters and using the following approximation:

$$
t_{\text {em }} \simeq t_{\text {arr }}-x \sin \left[\frac{2 \pi}{P_{\text {orb }}}\left(t_{\text {arr }}-T^{*}\right)\right],
$$

where $x=a \sin i / c$ is the projected semi-major axis in light-seconds, $T^{*}$ is the time of the ascending node passage at the beginning of the observation, and $t_{e m}$ and $t_{\text {arr }}$ are the emission and the arrival times, respectively. From the corrected time series we calculate the pulse phase delays fitting the pulse profiles, obtained from time intervals of length of the order of one orbital period, with one or more sinusoids (according to the harmonic content of the pulse profile) with fixed periods, and we plot the phase of the fundamental 
(and, when present, the phase of the harmonics) as a function of time to study the phase delays evolution.

The differential of expression (1) with respect to the orbital parameters allows to calculate the uncertainties, $\sigma_{\phi, o r b}$, on the phase delays induced by the uncertainties on the orbital parameters. Possible errors in the adopted values of the orbital parameters will indeed show up as a timing noise in the pulse phase delays of amplitude $\sigma_{\phi, o r b}$, and we sum in quadrature these uncertainties to the corresponding statistical uncertainties found from the fit of the pulse profile. This noise can be further reduced calculating the phase delays over intervals of length of the order of one orbital period, since the uncertainties induced by the errors on the orbital parameters are sinusoids of period $P_{o r b}$.

On the other hand, possible errors on the assumed position of the source in the sky will give a modulation of the phase delays with a period of 1 year. Since the X-ray observations span time intervals of a few tens of days at most (pulsations from accreting millisecond pulsars are usually observed during X-ray outbursts which last typically less than month), this induced modulation on the phase delays will appear like a linear trend or a parabola (the first orders of the series expansion of sinusoids with period 1 year). Therefore, the uncertainties in the source position can be considered like a systematic error on the linear correction to the spin period (linear term) and/or on the spin period derivative (parabolic term). This means that it is mandatory to have a good knowledge of the source position on the sky (such that is derived from optical or radio counterparts) in order to reduce these systematics.

In all our timing analyses we have included all these possible source of error, considering the uncertainties induced by errors in the orbital parameters as an additive noise in the pulse phase delays, and the uncertainties induced by errors in the source position as systematic uncertainties in the period and its derivative. In all the cases reported below, the systematics resulted much smaller than (or at most of the same order of magnitude of) the statistical errors on the linear and quadratic terms derived from the fit of the phase delays.

\section{IGR J00291+5934}

IGR J00291+5934 was discovered by the INTEGRAL satellite in December 2005, when it showed an X-ray outburst which lasted from December 3 to 21. X-ray pulsations were significantly detected only during the first 12 days of the outburst. With a spin period of $1.7 \mathrm{~ms}$ is the fastest among the known accreting millisecond pulsars and belongs to a binary system with orbital period $\sim 2.5 \mathrm{~h}$ [7]. Falanga et al. [8] report for this source the presence of a constant spin-up of $\sim 8 \times 10^{-13} \mathrm{~Hz} / \mathrm{s}$. Burderi et al. [6] re-analysed these data and, in particular, fitted the phase delays vs. time with physical models taking into account the observed decrease of the X-ray flux as a function of time during the X-ray outburst, in order to get a valuable estimate of the mass accretion rate onto the compact object. In fact, in the hypothesis the the spin-up of the source is caused by the accretion of matter and angular momentum from a Keplerian accretion disk, the mass accretion rate, $\dot{M}$ onto the neutron star can be calculated by the simple relation $2 \pi I \dot{V}=\dot{M}(G M R)^{1 / 2}$, where $I$ is the moment of inertia of the neutron star, $\dot{v}$ the spin 
frequency derivative, $G$ the Gravitational constant, $M$ the mass of the compact object, $R$ the accretion radius, and $(G M R)^{1 / 2}$ the Keplerian specific angular momentum at the accretion radius. Since we are neglecting any threading effect of the magnetic field in the accretion disk outside the accretion radius, the estimate of $\dot{M}$ derived in this way should be considered as a lower limit.

Because the X-ray flux, which is assumed to be a good tracer of the mass accretion rate, is observed to decrease along the outburst, this has to be included in the relation in order to obtain the correct value of the mass accretion rate at the beginning of the outburst as well as its temporal evolution (note that the accretion radius also depends on the mass accretion rate, $R \propto \dot{M}^{\alpha}$ where $\alpha$ is usually assumed to be $2 / 7$, and therefore is a function of time). From an analysis of the X-ray light curve, we concluded that the flux was, in good approximation, decreasing linearly with time, and adopted the following dependence: $\dot{M}(t)=\dot{M}_{0}\left[1-\left(t-T_{0}\right) / t_{B}\right]$, where $T_{0}$ is the time at the beginning of the observation (Dec 7), and $t_{B}=8.4$ days. We can therefore derive the following expression for the expected evolution of the phase delays vs. time:

$$
\phi=-\phi_{0}-\Delta v_{0}\left(t-T_{0}\right)-\frac{1}{2} \dot{v}_{0}\left(t-T_{0}\right)^{2}\left[1-\frac{(2-\alpha)\left(t-T_{0}\right)}{6 t_{B}}\right],
$$

where $\phi_{0}$ is a constant, $\Delta v_{0}$ is the linear correction to the value of the spin frequency adopted to produce the pulse profiles, and $\dot{v}_{0}$ is the frequency derivative at $t=T_{0}$. For the fit we used three possible values for $\alpha$, i.e. a) the standard $\alpha=2 / 7$ which corresponds to assuming that the accretion radius is proportional to the Alfven radius, b) $\alpha=0$ which corresponds to an accretion radius equal to the corotation radius (the radius at which the Keplerian frequency equals the neutron star spin frequency, that is the maximum radius at which accretion can occur), and c) $\alpha=2$ which corresponds to a simple parabolic function, that is to a constant mass accretion rate. For all the assumed values of $\alpha$ we obtained acceptable fits, and we have calculated the lower limit to the mass accretion rate at the beginning of the outburst, that is obtained in the case $\alpha=0$ :

$$
\dot{M}_{-10}=5.9 \times \dot{v}_{-13} I_{45} m^{-2 / 3}
$$

where $\dot{M}_{-10}$ is $\dot{M}_{0}$ in units of $10^{-10} M_{\odot} \mathrm{yr}^{-1}, \dot{v}_{-13}$ is $\dot{v}_{0}$ in units of $10^{-13} \mathrm{~s}^{-2}, I_{45}$ is $I$ in units of $10^{45} \mathrm{~g} \mathrm{~cm}^{2}$, and $m$ is the mass of the neutron star in units of $\mathrm{M}_{\odot}$. We adopt the FPS equation of state for the neutron star matter for $m=1.4$ and the spin frequency of IGR J00291+5934 which gives $I_{45}=1.29$ and $R_{N S}=1.14 \times 10^{6} \mathrm{~cm}$ (see e.g. [9]). Pulse phase delays and the corresponding best fit functions for all the three values of $\alpha$ are shown in Fig. 1 .

From the fitting of the phase delays with these relations we find $\dot{v}_{-13}=11.7$, and a lower limit to the mass accretion rate of $\dot{M}_{-10} \sim 70 \pm 10$ (case $\alpha=0$ ). This would correspond to a bolometric luminosity of $\sim 7 \times 10^{37} \mathrm{ergs} / \mathrm{s}$. This is about an order of magnitude higher than the X-ray luminosity inferred from the observed X-ray flux and assuming a distance of $5 \mathrm{kpc}$. Burderi et al. [6] have argued that, since the pulse profile is very sinusoidal with negligible harmonic content, we probably just see only one of the two emitting polar caps, and therefore the observer intercepts just half of the total emitted $\mathrm{X}$-ray luminosity. In this way, we can reduce the discrepancy between the bolometric 


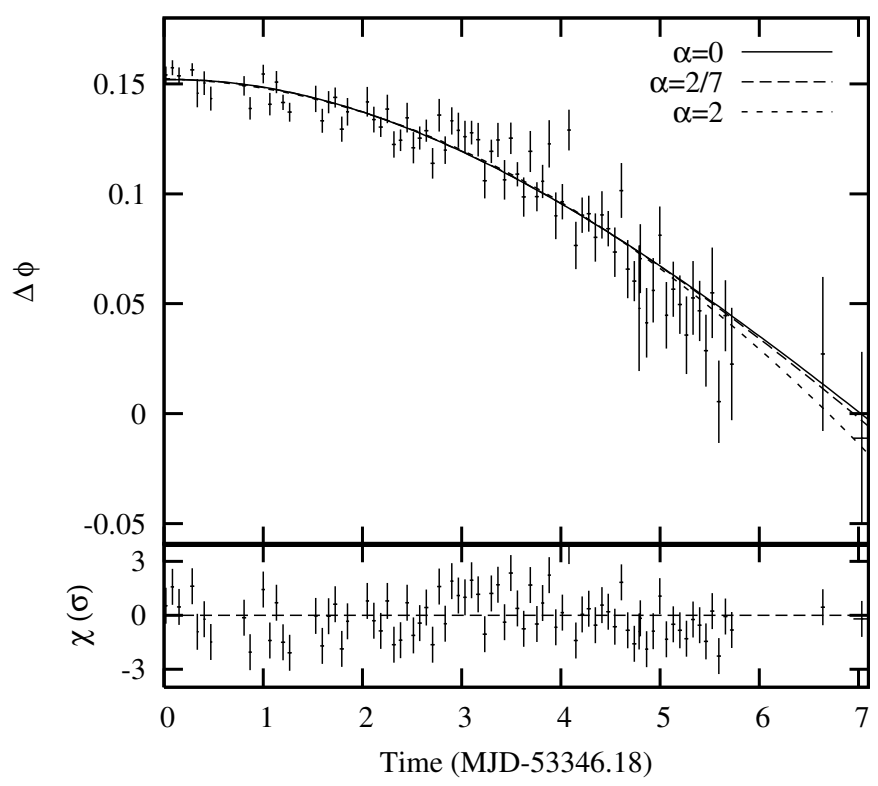

FIGURE 1. Pulse phases computed folding at the spin period reported in Table 1 and plotted versus time together with the best fit curves (upper panel) and residuals in units of $\sigma$ with respect to the model with $\alpha=2 / 7$ (lower panel).

luminosity inferred from the mass accretion rate and the observed X-ray luminosity, but still we need to place the source to a quite large distance of $7.4-10.7 \mathrm{kpc}$ (note that $10 \mathrm{kpc}$ is close to the edge of the Galaxy in the direction if IGR J00291+5934). Other possible explanations for the discrepancy between the mass accretion rate inferred by the timing and the observed X-ray luminosity can be that the energy released by accretion is not completely converted into X-ray luminosity, but a fraction of this is released in other energy bands or other emission mechanisms, or that the moment of inertia is smaller than the assumed value. As soon as we will have a direct, independent, estimate of the distance to the source, we will have very important information on the $\dot{M}$ - X-ray luminosity relation and/or on the physical parameters of the neutron star.

\section{SAX J1808.4-3658}

SAX J1808.4-3658 is the only one among accreting millisecond pulsars to have shown more than one X-ray outburst during the RXTE era; it goes into outburst roughly every two years (in 1998, 2000, 2002, and 2005 up to date). We have performed a timing analysis of the 2002 outburst, which lasted about 40 days from October 15 to November 26, one of the most extensively covered by RXTE observations (see details in [10]). In this case, the pulse profile shows the presence of a significant first harmonic, and we therefore studied both the phase delays of the fundamental and the phase delays of the harmonic as a function of time. If we look at the phase delays derived from the 

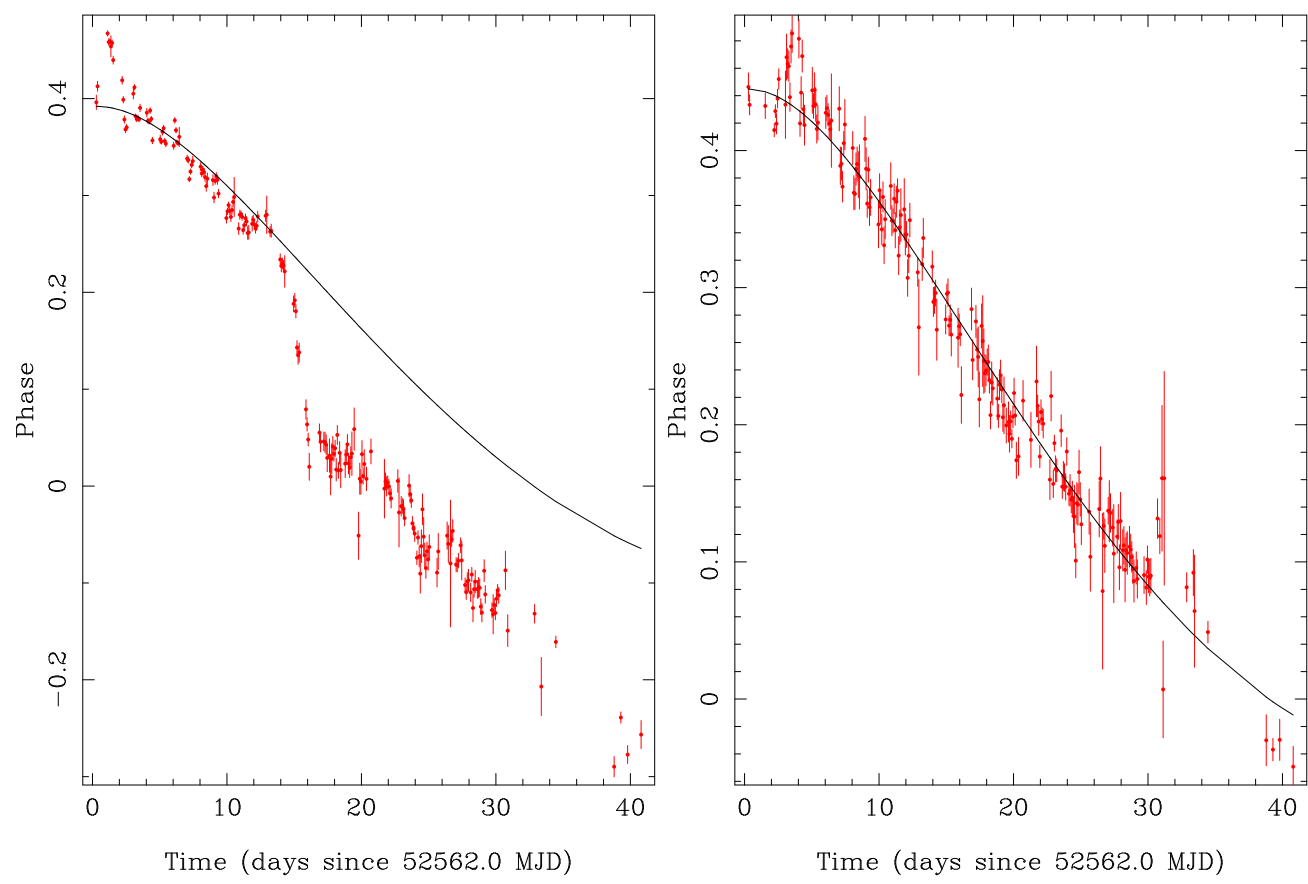

FIGURE 2. Left: Phase vs. time for the fundamental of the pulse frequency of SAX J1808.4-3658. Right: Phase vs. time for the first harmonic of the pulse frequency of SAX J1808.4-3658. On top of the data, the best fit function (the sum of eq. (4) and a quadratic term) is plotted as a solid line.

fundamental in Fig. 2. we can see a very puzzling behavior, since a rather fast phase shift is present at day 14 from the beginning of the outburst. Interestingly, as it can be easily seen from the X-ray light curve of the outburst, day 14 corresponds to a change in the steepness of the exponential decay with time of the X-ray flux. On the other hand, the phase delays of the harmonic do not show any evidence of this phase jump. This is not an effect of the worse statistics we have for the phase delays derived from the harmonic, which of course show larger error bars. We have to conclude that the phase jump in the fundamental is not related to a intrinsic spin variation (which would have affected the whole pulse profile), but is instead caused by a change of the shape of the pulse profile (probably caused by the same mechanism causing the increase of the steepness of the exponential decay of the X-ray flux).

Given the regular behavior of the phase delays of the harmonic, we tried to fit these to an appropriate model. As in the case of IGR J00291+5934, we considered a varying with time mass accretion rate, that in this case is exponentially decreasing during the outburst: $\dot{M}(t)=\dot{M}_{0} \exp \left(t-T_{0}\right) / \tau$, where $\tau=9.27$ days can be derived from a fit of the (first 14 days) light curve. Again we can derive the expected variation of the phase delays for the case of an exponentially decreasing mass accretion rate:

$$
\phi(t)=\phi_{0}-B\left(t-T_{0}\right)-C \exp \left(t-T_{0}\right) / \tau,
$$

where $C=1.067 \times 10^{-4} I_{45}^{-1} P_{-3}^{1 / 3} m^{2 / 3} \tau^{2} \dot{M}_{-10}, P_{-3}$ is the spin period in millisecond, and $B=\Delta v_{0}+C / \tau$. However, we obtained a poor fit both using the expression above or using a simple parabolic trend. Indeed, with the model of eq. 4 we can obtain a good fit 
of the first 14 days of the outburst, but, with respect to this fit, we observe a flattening of the phase delays after day 14. To describe this flattening we therefore added to eq. 4 a quadratic term corresponding to a constant spin-down. The best fit function is plotted on top of the data in Fig. 2 .

From this best fit we can derive a spin-up at the beginning of the outburst of $\dot{v}_{0} \sim 4.4 \times 10^{-13} \mathrm{~Hz} / \mathrm{s}$, corresponding to a mass accretion rate of $\dot{M}_{-10}=18$, and a (marginally significant 1 ) constant spin-down of $\dot{v}_{s d} \sim-7.6 \times 10^{-14} \mathrm{~Hz} / \mathrm{s}$. In the case of SAX J1808.4-3658 the distance to the source is known and is about $3.5 \mathrm{kpc}$ [11]; therefore we can check if also in this case there is a discrepancy between the mass accretion rate inferred from the timing results and the observed X-ray luminosity. We still find a discrepancy, but, in this case, the mass accretion rate inferred from timing is only a factor of 2 larger than the X-ray luminosity, since this is about $1 \times 10^{37} \mathrm{ergs} / \mathrm{s}$ (see [10]). The spin-down observed at the end of the outburst can be explained, for instance, by a threading of the accretion disk by the neutron star magnetic field outside the accretion radius. Of course, in agreement with what we observe, we expect that such a threading effect will be observable at the end of the outburst, when the mass accretion rate significantly decreases (see [12]). We can therefore evaluate the magnetic moment, $\mu$, of SAX J1808.4-3658 from our measured value of the spin-down, using the relation $\mu^{2} /\left(9 R_{C O}^{3}\right)=2 \pi I \dot{v}_{s d}$, where $R_{C O}$ is the corotation radius. The magnetic field found in this way is $B \sim(3.5 \pm 0.5) \times 10^{8}$ Gauss, perfectly in agreement with previous constraints [13].

\section{XTE J1814-338}

XTE J1814-338 was discovered in 2003 by RXTE [14]; the X-ray outburst started on June 5 and lasted about 53 days. The spin period is $\sim 3.14 \mathrm{~ms}$ and the binary orbital period, $P_{\text {orb }}=4.275 \mathrm{~h}$, is the largest in the sample of known accreting millisecond pulsars. We have performed a timing analysis of this source (see [15], and the paper by Papitto et al. included in this volume) finding that the neutron star shows a global spin-down, $\dot{v}_{s d} \sim(-6.7 \pm 0.7) \times 10^{-14} \mathrm{~Hz} / \mathrm{s}$, during all the outburst. Again this source shows a puzzling behavior of the phase delays; for this source the harmonic contain in the pulse profile is quite high. In particular we detect the fundamental and the first harmonic, whose amplitudes are both quite large. We have therefore plotted the phase delays of both the fundamental and the harmonic, finding in this case that both show the same trend when plotted vs. time. This trend is approximately parabolic and showing a global spin-down of the pulsar, but superposed to this general trend we find oscillations of the phase delays.

Differently from previous cases, the X-ray flux in this source does not monotonically decay during the outburst; instead the flux is observed to oscillate around a mean value during the first 30 days of the outburst, and then it fastly decays to quiescence. We find that the oscillations observed in the phase delays of the fundamental and the harmonic

${ }^{1}$ Due to the uncertainty in the modelization of the X-ray flux behavior vs. time in the second half of the outburst 
are very well anticorrelated with the oscillations present in the X-ray flux. We have therefore interpreted, similarly to the case of the phase shift observed in SAX J1808.43658 , these oscillations as phase shifts induced by small movements of the magnetic field footpoints in the neutron star surface driven by variations of the X-ray flux.

Also in this case, we can try to get an estimate of the neutron star magnetic field from the observed global spin-down trend and using the threading of the accretion disk model (as in [12]). We get a quite large value for the magnetic field of $\sim 8 \times 10^{8}$ Gauss.2]

\section{XTE J0929-314}

XTE J0929-314 is a high-latitude source and was discovered by RXTE in 2002, when it showed an X-ray outburst which started on May 2 and lasted for about 53 days. Galloway et al. [16] provided an orbital solution for this source, reporting a quite short period of $\sim 44 \mathrm{~min}$. With a spin period of $\sim 5.4 \mathrm{~ms}$ this is the slowest among the known sample of accreting millisecond pulsars. They also performed a timing analysis of the pulse phase delays, showing that the source underwent a steady spindown while accreting of $\dot{v}=-9.2(4) \times 10^{-14} \mathrm{~Hz} / \mathrm{s}$. We have re-analysed these data, using an improved source position on the sky, and basically confirm the already reported results, although with a revised spin-down rate of $\dot{v}=-5.5(4) \times 10^{-14} \mathrm{~Hz} / \mathrm{s}$.

Although the timing results seem quite simple in this case, indeed XTE J0929-314 shows the most puzzling behavior with respect all the sample of accreting millisecond pulsars discussed here. In fact, as in the case of IGR J00291+5934, XTE J0929-314 shows an almost linear decrease of the X-ray flux during the outburst, with a decay time $t_{B} \simeq 58.5$ days. This means that, along the outburst, the expected spin-up should decrease, and the global derivative of the spin frequency (that is the sum of the spin-up and spin-down rates) should show an increasing global spin-down along the outburst. However, the pulse phase delays do not show any increasing spin-down, since the best fit suggests a constant (or at most decreasing) spin-down. If the decreasing of the X-ray flux does not affect the behavior of the phase delays, this means that the corresponding spin-up rate should be always negligible with respct to the observed spin-down, i.e. $\dot{v}_{s u}<<-\dot{v}_{s d} \sim 5.5 \times 10^{-14} \mathrm{~Hz} / \mathrm{s}$. If we assume, in agreement with the request above, that the spin-up is at least a factor of 5 lower than the spin-down rate, we find a corresponding mass accretion rate of $\dot{M}<6 \times 10^{-11} \mathrm{M}_{\odot} / \mathrm{yr}$, which would correspond to a quite low bolometric luminosity of $<6 \times 10^{35} \mathrm{ergs} / \mathrm{s}$. If we compare this luminosity with the observed X-ray luminosity, $L_{X} \sim 1.0 \times 10^{37} \mathrm{~d}_{5 k p c}^{2}$ ergs/s where $\mathrm{d}_{5 k p c}^{2}$ is the distance to the source in units of $5 \mathrm{kpc}$, we find an upper limit to the source distance of about $1.2 \mathrm{kpc}$, that is less than the lower limit of $5 \mathrm{kpc}$ derived by the expected secular mass accretion rate (driven by gravitational radiation) and a supposed outburst recurrence time $>6.5 \mathrm{yr}[16]$.

A distance of $\sim 1 \mathrm{kpc}$ is unlikely to be correct, although we have to note that

\footnotetext{
2 The magnetic field has been evaluated using for the average mass accretion rate during the first 35 days of the outburst, when the mass accretion rate can be considered almost constant, the value $\sim 5.4 \times 10^{-10}$ $\mathrm{M}_{\odot} / \mathrm{yr}$, and assuming a distance to the source of $8 \mathrm{kpc}$.
} 
TABLE 1. Summary of timing results for our sample of accreting millisecond pulsars

\begin{tabular}{lrrr}
\hline Source & \multicolumn{1}{c}{$\mathbf{P}_{\text {orb }}(\mathbf{h})$} & \multicolumn{1}{c}{$\mathbf{P}_{\text {spin }}(\mathbf{m s})$} & \multicolumn{1}{c}{$\dot{v}(\mathbf{H z} / \mathbf{s})$} \\
\hline IGR J00291+5934 & $2.456692(2)$ & $1.66974977466(5)$ & $1.2(2) \times 10^{-12}$ \\
SAX J1808.4-3658 & $2.01365469(3)$ & $2.4939197632(4)$ & $4.4(8) \times 10^{-13}$ \\
SAX J1808.4-3658 & & & $-7.6(1.5) \times 10^{-14}$ \\
XTE J1814-338 & $4.27464525(5)$ & $3.1811056697(1)$ & $-6.7(7) \times 10^{-14}$ \\
XTE J0929-314 & $0.7263183(8)$ & $5.4023317862(3)$ & $-5.5(4) \times 10^{-14}$ \\
\hline
\end{tabular}

XTE J0929-314 is a high latitude source, and therefore the closer is the source, the smaller will be the height of the source above the Galactic plane. Otherwise, the reason of this discrepancy may be in the used model for the threading (spin-down) torque; in most of the models this depends only on the magnetic field strength and should therefore remain constant along an X-ray outburst. However, the pulse phase delays seem to suggest that the spin-down in XTE J0929-314 may decrease at the end of the outburst. We note that results of MHD simulations on the interaction between the accretion disc and the magnetosphere of a NS in the propeller regime (i.e. when the accretion radius is larger than corotation radius) presented by [17]; see also [18]) show that the spin down torque resulting from this interaction may decrease with decreasing accretion rate, with the material torque owing to the accreted matter relegated to a marginal role in building the overall torque. This behavior is related to the weaker coupling between the magnetosphere and the disc matter corresponding to a lower accretion rate, which has the effect to weaken the toroidal component of the magnetic field in the magnetosphere, which is the one responsible for the spinning down of the pulsar. We therefore need a correct modelization of the spin-down torque in order to correctly evaluate the upper limit on the mass accretion rate from timing.

\section{DISCUSSION AND CONCLUSIONS}

In this paper we review the results of a timing analysis performed over a sample of accreting millisecond pulsars (a summary of these results is shown in Tab. 1). We have showed that a few accreting millisecond pulsars, which are supposed to accrete from a Keplerian accretion disk, show steady spin-down while accreting. The only (thus far) available explanation for this is in terms of the magnetic field - accretion disk interaction, that is a threading of the accretion disk by the magnetic field outside the accretion radius. However, this predicts a quite low luminosity in the case of XTE J0929-314, and therefore a quite small distance to the source. Independent measurements of the distance to XTE J0929-314 will give important information on the torque acting on the neutron star and its response. Most of our results are puzzling but many of them are exactly as expected: IGR J00291+5934 shows the strongest spin-up, in agreement with the fact that it is the fastest accreting millisecond pulsars; slower pulsars show less spin-up or spin-down. In SAX J1808.4-3658 we observe a shift in phase of the fundamental at day 14 , the same day at which is observed a steepness of the exponential decay of the X-ray flux, and again around that day there seems to be a change from spin-up to spin-down 
of the pulsar. These facts are in agreement with a scenario where some sort of ejection mechanism becomes important in the disk when the mass accretion rate is sufficiently low; this explains the increased steepness in the flux decay and the possible change from global spin-up to global spin-down. This may also be responsible of movements of the footpoints of the magnetic field onto the neutron star surface, and therefore of the change of the shape of the pulse profile, that is observed as a shift in phase of the fundamental, although the detailed mechanism is not clear yet.

\section{ACKNOWLEDGMENTS}

This work was supported by the Ministero della Istruzione, della Università e della Ricerca (MIUR), national program PRIN2005 2005024090_004.

\section{REFERENCES}

1. Bhattacharya D., \& van den Heuvel E. P. J., Physics Reports, 203, 1 (1991).

2. Wijnands R., \& van der Klis M., Nature, 394, 344 (1998).

3. Chakrabarty D., \& Morgan E. H., Nature, 394, 346 (1998).

4. Wijnands R., To appear in Nova Science Publishers (NY) volume "Pulsars New Research" (2005).

5. Galloway D. K., to be published in the Proceedings of the 7th Pacific Rim Conference on Stellar Astrophysics astro-ph/0608540 (2006).

6. Burderi L., Di Salvo T., Lavagetto G., et al. , ApJ, 657, 961 (2007).

7. Galloway D. K., Markwardt C. B., Morgan E. H., et al., ApJ, 622, L45 (2005).

8. Falanga M., Kuiper L., Poutanen J., et al. , $A \& A, 444,15$ (2005).

9. Cook G. B., Shapiro, S. L., Teukolsky, S. A., ApJ, 424, 823 (1994)

10. Burderi L., Di Salvo T., Menna M. T., Riggio A., Papitto A., ApJ, 653, L133 (2006)

11. Galloway D. K., \& Cumming A., ApJ, 652, 559 (2006)

12. Rappaport S. A., Fregeau J. M., Spruit H., ApJ, 606, 436 (2004)

13. Di Salvo T., Burderi L., A\&A, 397, 723 (2003)

14. Markwardt C. B., \& Swank J. H., IAUC, 8144, 1 (2003)

15. Papitto A., Di Salvo T., Burderi L., Menna M. T., Lavagetto G., Riggio A., MNRAS, 375, 971 (2007)

16. Galloway D. K., Chakrabarty D., Morgan E. H., Remillard R. A., ApJ, 576, L137 (2002)

17. Romanova M. M., Ustyugova G. V., Koldoba A. V., Lovelace R.V.E., ApJ, 616, L151 (2004)

18. Romanova M. M., Ustyugova G. V., Koldoba A. V., Lovelace R.V.E., ApJ, 635, L165 (2005). 